\title{
Article \\ The Use of Trapezoidal Oriented Fuzzy Numbers in Portfolio Analysis
}

\author{
Anna Lyczkowska-Hanćkowiak (D)
}

Citation: Łyczkowska-Hanćkowiak, A. The Use of Trapezoidal Oriented Fuzzy Numbers in Portfolio Analysis. Symmetry 2021, 13, 1722. https:// doi.org/10.3390/sym13091722

Academic Editors: Kóczy T. László and István A. Harmati

Received: 20 August 2021

Accepted: 14 September 2021

Published: 17 September 2021

Publisher's Note: MDPI stays neutral with regard to jurisdictional claims in published maps and institutional affiliations.

Copyright: (C) 2021 by the author. Licensee MDPI, Basel, Switzerland. This article is an open access article distributed under the terms and conditions of the Creative Commons Attribution (CC BY) license (https:// creativecommons.org/licenses/by/ $4.0 /)$.
Institute of Economy and Finance, WSB University in Poznan, ul. Powstańców Wielkopolskich 5, 61-895 Poznań, Poland; anna.lyczkowska-hanckowiak@wsb.poznan.pl

\begin{abstract}
Oriented fuzzy numbers are a convenient tool to manage an investment portfolio as they enable the inclusion of uncertain and imprecise information about the financial market in a portfolio analysis. This kind of portfolio analysis is based on the discount factor. Thanks to this fact, this analysis is simpler than a portfolio analysis based on the return rate. The present value is imprecise due to the fact that it is modelled with the use of oriented fuzzy numbers. In such a case, the expected discount factor is also an oriented fuzzy number. The main objective of this paper is to conduct a portfolio analysis consisting of the instruments with the present value estimated as a trapezoidal oriented fuzzy number. We consider the portfolio elements as being positively and negatively oriented. We test their discount factor. Due to the fact that adding oriented fuzzy numbers is not associative, a weighted sum of positively oriented discount factors and a weighted sum of negatively oriented factors is calculated and consequently a portfolio discount factor is obtained as a weighted addition of both sums. Also, the imprecision risk of the obtained investment portfolio is estimated using measures of energy and entropy. All theoretical considerations are illustrated by an empirical case study.
\end{abstract}

Keywords: oriented fuzzy number; imprecision; present value; discount factor; portfolio; finance

\section{Introduction}

Imprecision is one of the characteristic features of information on financial markets. According to the uncertainty theory, any unknown future state of affairs is uncertain [1,2]. Uncertainty arises from our ignorance of the future state of affairs. This means that it can be modelled with a certain probability, as long as we are able to indicate a specific time at which the considered state of affairs will be known to the observer [3-9]. This was initially conceived by Kolmogorov [5,6] and is called "Kolmogorov's postulate". Imprecision is perceived as a combination of ambiguity and indistinctness of information [10]. Ambiguity is understood as a lack of a clear recommendation between one alternative among various others. Indistinctness is interpreted as a lack of explicit distinction between recommended and unrecommended alternatives. Modelling the imprecision by its membership function of the fuzzy set is a commonly used method [11]. In this paper, we describe imprecision using oriented fuzzy numbers. In portfolio analyses, we use uncertain and imprecise information about the financial market.

A security is an authorization to receive future financial income that is payable to a specified maturity. The value of this income is described as an anticipated future value of capital. The present value (PV) is defined as a current equivalent of a payment available at a stated time in the future [12]. It is assumed that the present value of future cash flows is an approximated value and therefore it can be modelled by fuzzy numbers. In this paper, PV is modelled by oriented fuzzy numbers (OFN) defined in [13]. In recent years, OFNs have been increasingly used to describe and analyze economic [14,15], financial [16-25] and decision-making [26-33] problems. The application of OFNs in financial analysis may minimize imprecision risk, which was presented, for instance, in [32]. The family of 
all oriented fuzzy numbers has a symmetry axis that is equal to the family $\mathrm{R}$ of all real numbers, which was discussed in detail in [32,34].

The base of any security assessment is the return rate, generally defined as an arbitrary non-increasing function of present value and a non-decreasing function of future value. An arbitrary, finite set of securities will be called a financial portfolio. After Markowitz, we also assume simple return rates, which means that the current value is positive and the future value is a random variable with a normal distribution [35]. It allows for some of the parameters, considered in existing portfolio theory, such as return rate or present value [36-38] or probability distribution parameters [39], to be fuzzy. Fuzzy systems are increasingly used in portfolio analysis [40-45]. A detailed evolution of research was presented in $[17,32]$.

In financial arithmetic, fuzzy numbers appeared in 1987 due to Buckley [46]. The definition, which was proposed by Ward [47], was generalized to the case of imprecisely assessed postponement [48], fuzzy nominal interest rate [49], future cash flow described by a fuzzy variable [50], and to the case when future cash flow can be treated as a fuzzy probabilistic set [51]. The current fuzzy value was axiomatically defined by Calzi [52], while in Piasecki [53] the fuzzy PV was estimated based on the current quoted price of a financial asset. More detailed elaboration on that topic can be found in [17,32], among others.

A portfolio with a trapezoidal fuzzy PV was investigated, for instance, in [45]. In financial portfolio management, utilizing oriented fuzzy numbers is more useful than utilizing fuzzy numbers. We use trapezoidal oriented fuzzy numbers in the portfolio analysis because performing summation operations on them is much easier than on oriented fuzzy numbers. Oriented fuzzy numbers can be approximated by trapezoidal fuzzy numbers, as shown in [54]. Operations on oriented fuzzy numbers are much more computationally complicated, as can be seen in [17]. In [21], Piasecki and Łyczkowska-Hanćkowiak showed a universal method of representing Japanese candlesticks with oriented fuzzy numbers. In our paper, we investigate portfolios with PVs described by oriented fuzzy numbers. Moreover, a portfolio analysis based on a fuzzy discount factor is simpler than a portfolio analysis based on a return rate. For this reason, here we use a discount factor evaluated by oriented fuzzy numbers as defined in [25]. This paper presents an original method of using the imprecision expected discount factor in the portfolio analysis. We also show that portfolio diversification may lower uncertainty risk and imprecision risk. The addition of oriented fuzzy numbers is not associative. Therefore, we calculate the weighted sum of positively oriented discount factors and the sum of negatively oriented discount factors separately. Then, the portfolio discount factor is obtained by the weighted addition of these sums. Such a procedure for determining the discount factor of the portfolio is justified by economic premises. The criterion of the maximization of the expected return rate is replaced by the criterion of the minimalization of the expected discount factor. In decision analysis, a convenient tool for measuring the indistinctness risk is the entropy measure. In each of the considered cases, a return rate is a function of future value, which is uncertain by its nature. This uncertainty results from the ignorance of the future state of matters. This ignorance causes the investor to be unsure of future gains or losses. An increase in uncertainty may increase the uncertainty risk, which is the risk of making an incorrect financial decision. An increase in ambiguity means an increase in the amount of highly recommended alternative information about the state of affairs. This increase in the ambiguity of imprecise expected discount factors indicates that the number of alternative decisions that can be selected will be greater. This implies an increased ambiguity risk, i.e., selecting an incorrect assessment from among the recommended ones. This can cause a decision that will result in less than the maximum profit. Ambiguity and uncertainty risk have the same description of the dangers caused by risks taken and the effects of decisions made but they differ in their causes. For this reason, both types of risk should be assessed by different methods. We estimate the ambiguity risk of encumbering the imprecise expected discount factor with the energy measure. An increase in the indistinctness of the imprecise expected discount factor suggests that the differences between the 
recommended and unrecommended decision alternatives are harder to differentiate. This suggests that the indistinctness risk may increase. This means that we can choose an option that is not recommended. We estimate the indistinctness risk of the imprecise expected discount factor using the entropy measure. The risk of imprecision is the combination of the ambiguity and indistinctness risk. To assess the ambiguity and indistinctness risk, we use the energy measure and entropy measure, respectively. The main objective of this paper is to prove that it is possible to effectively manage a financial asset portfolio while accounting for both imprecision and uncertainty of information about the market. By using OFNs in the analysis, we minimize the imprecision risk.

The paper is organized as follows. Section 2 outlines trapezoidal fuzzy numbers and their basic properties as a theoretical background for our future considerations. Section 3 contains the evaluation of imprecision for oriented fuzzy numbers. In Section 4, we introduce the notion of imprecisely estimated PVs, which are evaluated using trapezoidal oriented fuzzy numbers. We use the obtained model in Section 5, where the expected discount factor is researched. In Section 6, we compare the original method for determining multi-asset portfolios with trapezoidal oriented fuzzy PVs. Our research is illustrated by a case study. This case study sufficiently explains the proposed method of portfolio evaluation. Finally, Section 7 concludes the article and summarizes the main findings.

\section{Trapezoidal Oriented Fuzzy Numbers-Basic Facts}

The family of all fuzzy subsets in the real line $\mathbb{R}$ we denote by $\mathscr{F}(\mathbb{R})$. Any fuzzy subset $\mathscr{A} \in \mathscr{F}(\mathbb{R})$ is described by its membership function $\mu_{A} \in[0,1]^{\mathbb{R}}$ as a set of ordered pairs:

$$
\mathscr{A}=\left\{\left(x, \mu_{A}(x)\right) ; x \in \mathbb{R}\right\}
$$

Fuzzy subset $\mathscr{A}$ may be characterized by its support closure $[\mathscr{A}]_{0^{+}}$, given in the following way:

$$
[\mathscr{A}]_{0^{+}}=\lim _{\alpha \rightarrow 0^{+}}\left\{x \in \mathbb{R}: \mu_{A}(x) \geq \alpha\right\} .
$$

The core $\operatorname{Core}(A)$ is defined by the formula:

$$
\operatorname{Core}(A)=\left\{x \in \mathbb{X}: \mu_{A}(x)=1\right\}
$$

A fuzzy number (FN) is usually defined as a fuzzy subset of the real line $\mathbb{R}$. The most general definition of FN is given as follows:

Definition 1 [55]. The fuzzy number is such a fuzzy subset $\mathscr{L} \in \mathscr{F}(\mathbb{R})$ with a bounded support closure $[\mathscr{L}]_{0^{+}}$that it is represented by its upper semi-continuous membership function $\mu_{L} \in[0 ; 1]^{\mathbb{R}}$, satisfying the conditions:

$$
\begin{gathered}
\exists_{x \in \mathbb{R}} \mu_{L}(x)=1 \\
\forall_{(x, y, z) \in \mathbb{R}^{3}} x \leq y \leq z \Longrightarrow \mu_{L}(y) \geq \min \left\{\mu_{L}(x) ; \mu_{L}(z)\right\}
\end{gathered}
$$

The set of all FNs we denote by the symbol $\mathbb{F}$. Any FN may be represented in the following way:

Theorem 1 [56,57]. For any FN $\mathscr{L}$ there exists such a non-decreasing sequence $(a, b, c, d) \subset \mathbb{R}$ that $\mathscr{L}\left(a, b, c, d, L_{L}, R_{L}\right)=\mathscr{L} \in \mathscr{F}(\mathbb{R})$ is determined by its membership function $\mu_{L}\left(\cdot \mid a, b, c, d, L_{L}, R_{L}\right) \in[0,1]^{\mathbb{R}}$ described by the identity:

$$
\mu_{L}\left(x \mid a, b, c, d, L_{L}, R_{L}\right)=\left\{\begin{array}{c}
0, x \notin[a, d] \\
L_{L}(x), x \in[a, b] \\
1, x \in[b, c] \\
R_{L}(x), x \in[c, d]
\end{array}\right.
$$


where the left reference function $L_{L} \in[0,1]^{[a, b]}$. and the right reference function $R_{L} \in[0,1]^{[c, d]}$ are upper semi-continuous monotonic ones meeting the condition:

$$
[\mathscr{L}]_{0^{+}}=[a, d] .
$$

The FN $\mathscr{L}\left(a, a, a, a, L_{L}, R_{L}\right)=\llbracket a \rrbracket$ represents the real number $a \in \mathbb{R}$, i.e., $\mathbb{R} \subset \mathbb{F}$.

The notion of ordered FNs is introduced by Kosiński et al. in [58]. For formal reasons, Kosiński's theory is revised in [13]. In the revised theory, the notion of ordered FNs is narrowed down to the notion of oriented FNs (OFN), defined as follows:

Definition 2 [13]. For any monotonic sequence $(a, b, c, d) \subset \mathbb{R}$, an oriented fuzzy number $\overleftrightarrow{\mathscr{L}}\left(a, b, c, d, S_{L}, E_{L}\right)=\overleftrightarrow{\mathscr{L}}$ is the pair of orientation $\overrightarrow{a, d}=(a, d)$ and $F N \mathscr{L} \in \mathbb{F}$ is described by membership of the function $\mu_{L}\left(\cdot \mid a, b, c, d, S_{L}, E_{L}\right) \in[0,1]^{\mathbb{R}}$, given by the identity:

$$
\mu_{L}\left(x \mid a, b, c, d, S_{L}, E_{L}\right)=\left\{\begin{array}{c}
0, x \notin[a, d] \equiv[d, a], \\
S_{L}(x), x \in[a, b] \equiv[b, a], \\
1, x \in[b, c] \equiv[c, b], \\
E_{L}(x), x \in[c, d] \equiv[d, c],
\end{array}\right.
$$

where the starting function $S_{L} \in[0,1]^{[a, b]}$. and the ending function $E_{L} \in[0,1]^{[c, d]}$ are upper semi-continuous monotonic ones meeting condition (7).

In Equation (8), we use modified interval notation, often used in the theory of oriented fuzzy numbers. The notation $\mathscr{I} \equiv \mathscr{K}$ means that "the interval $\mathscr{I}$ may be equivalently replaced by the interval $\mathscr{K}^{\prime \prime}$. The relationships between FNs, ordered FNs, and OFNs are discussed in detail in [34].

The symbol $\mathbb{K}$ denotes the space of all OFNs. If $a<d$, then OFN $\overleftrightarrow{\mathscr{L}}\left(a, b, c, d, S_{L}, E_{L}\right)$ has a positive orientation $\overrightarrow{a, d}$. It informs us about the possibility of an increase in the approximated number. If $a>d$, then $\operatorname{OFN} \overleftrightarrow{\mathscr{L}}\left(a, b, c, d, S_{L}, E_{L}\right)$ has the negative orientation $\overrightarrow{a, d}$. It informs us about the possibility of a decrease in the approximated number. If $a=d$, then OFN $\overleftrightarrow{\mathscr{L}}\left(a, a, a, a, S_{L}, E_{L}\right)=\llbracket a \rrbracket$ describes the real number $a \in \mathbb{R}$.

Trapezoidal fuzzy numbers (TrOFNs) are a special case of OFNs.

Definition 3 [13]. For any monotonic sequence $(a, b, c, d) \subset \mathbb{R}, \operatorname{TrOFN} \overleftrightarrow{\operatorname{Tr}}(a, b, c, d)=\overleftrightarrow{\mathscr{T}}$ is OFN $\stackrel{\leftrightarrow}{\mathscr{T}} \in \mathbb{K}$, determined explicitly by its membership functions $\mu_{T} \in[0,1]^{\mathbb{R}}$ as follows:

$$
\mu_{T}(x)=\mu_{T r}(x \mid a, b, c, d)=\left\{\begin{array}{c}
0, x \notin[a, d] \equiv[d, a], \\
\frac{x-a}{b-a}, x \in[a, b] \equiv[a, b], \\
1, x \in[b, c] \equiv[c, b], \\
\frac{x-d}{c-d}, x \in[c, d] \equiv[c, d] .
\end{array}\right.
$$

The space of all trapezoidal oriented fuzzy numbers, TrOFNs, we denote as $\mathbb{K}_{T r}$. By $\mathbb{K}_{T r}^{+}$we denote the space of all TrOFNs that have a positive orientation and by $\mathbb{K}_{T r}^{-}$ we denote the space of all TrOFNs that have a negative orientation. A crisp number $a \in \mathbb{R}$, which is unoriented, is denoted as the following trapezoidal oriented fuzzy number $\overleftrightarrow{\operatorname{Tr}}(a, a, a, a)=\llbracket a \rrbracket$.

Let symbol $*$ denote any arithmetic operation defined in $\mathbb{R}$ and symbol $*$ denote an extension of an arithmetic operation $*$ to $\mathbb{K}$ [13]. Kosiński has defined arithmetic operators on ordered FNs in an intuitive way, but the addition of a dot product extended to the space $\mathbb{K}$ has a very high level of formal complexity [34]. Therefore, in many applications researchers limit their calculations to arithmetic operations determined in the space $\mathbb{K}_{T r}$. 
In line with Kosinski's approach, we can extend the basic arithmetic operators in the case of $\mathbb{K}_{\operatorname{Tr}}$ in such way that for any pair $(\overleftrightarrow{\operatorname{Tr}}(a, b, c, d), \stackrel{\leftrightarrow}{\operatorname{Tr}}(p-a, q-b, r-c, s-d)) \in \mathbb{K}_{\operatorname{Tr}}^{2}$ and $\beta \in \mathbb{R}$, arithmetic operations of extended sum $\boxplus$ and dot product $\square$ are defined as follows [34]:

$$
\begin{aligned}
& \begin{array}{c}
\overleftrightarrow{\operatorname{Tr}}(a, b, c, d) \boxplus \overleftrightarrow{\operatorname{Tr}}(p-a, q-b, r-c, s-d)= \\
=\left\{\begin{array}{l}
\overleftrightarrow{\operatorname{Tr}}(\min \{p, q\}, q, r, \max \{r, s\}),(q<r) \vee(q=r \wedge p \leq s), \\
\overleftrightarrow{\operatorname{Tr}}(\max \{p, q\}, q, r, \min \{r, s\}),(q<r) \vee(q=r \wedge p>s) .
\end{array}\right.
\end{array} \\
& \beta \boxminus \overleftrightarrow{\operatorname{Tr}}(a, b, c, d)=\overleftrightarrow{\operatorname{Tr}}(\beta \cdot a, \beta \cdot b, \beta \cdot c, \beta \cdot d)
\end{aligned}
$$

It is very easy to check that for any pair $(\overleftrightarrow{\operatorname{Tr}}(a, b, c, d), \overleftrightarrow{\operatorname{Tr}}(e, f, g, h)) \in \mathbb{K}_{T r}^{+} \times \mathbb{K}_{\operatorname{Tr}}^{+} \cup$ $\mathbb{K}_{T r}^{-} \times \mathbb{K}_{T r}^{-}$, we have:

$$
\overleftrightarrow{\operatorname{Tr}}(a, b, c, d) \boxplus \overleftrightarrow{\operatorname{Tr}}(e, f, g, h)=\overleftrightarrow{\operatorname{Tr}}(a+e, b+f, c+g, d+h)
$$

Any unary operator $G: \mathbb{R} \supset \mathbb{A} \longrightarrow \mathbb{R}$ may be extended in the case of TrOFNs. Using Kosiński's approach, we define an extended unary operator $\stackrel{\leftrightarrow}{G}: \mathbb{K}_{T r} \supset \mathbb{H} \longrightarrow \mathbb{K}$ as follows:

$$
\overleftrightarrow{\mathscr{L}}\left(G(a), G(b), G(c), G(d), S_{L}, E_{L}\right)=\overleftrightarrow{G}(\overleftrightarrow{\operatorname{Tr}}(a, b, c, d))
$$

where the starting function and the ending function are given by the formulas:

$$
\begin{aligned}
& \forall y \in[G(a), G(b)] S_{L}(y)=\frac{G^{-1}(y)-a}{b-a}, \\
& \forall y \in[G(c), G(d)] E_{L}(y)=\frac{G^{-1}(y)-d}{c-d} .
\end{aligned}
$$

OFN (12) is TrOFN if and only if its starting function and ending function are linear.

\section{Evaluation of Imprecision for Oriented Fuzzy Numbers}

Information imprecision we understand as a superposition of ambiguity and indistinctness of information [10]. Ambiguity is comprehended in such a way that neither option is unequivocally recommended. Indistinctness is comprehended in such a way that recommended and unrecommended alternatives are not clearly distinguishable.

The increase in OFN ambiguity implies a higher number of recommended alternatives. This increases the risk of choosing the wrong alternative from among the recommended ones. This can eventually lead to one making a decision that will result in the loss of ex-post chances. The possibility of this happening is called the ambiguity risk. Hence, an increase in the ambiguity of OFN causes an increase in the ambiguity risk. A convenient tool for measuring the oriented fuzzy number ambiguity is an extension of energy measure, defined by the following formula:

$$
d\left(\overleftrightarrow{\mathscr{L}}\left(a, b, c, d, L_{L}, R_{L}\right)\right)=\left|\int_{a}^{d} \mu_{L}\left(x \mid a, b, c, d, L_{L}, R_{L}\right) d x\right|
$$

This energy measure is an extension of the energy measure $d \in\left[\mathbb{R}_{0}^{+}\right]^{\mathbb{F}}$, defined for fuzzy numbers by de Luca and Termini [59]. In decision analysis, to measure the ambiguity risk is to exploit the energy measure. 
A comfortable instrument for measuring the indistinctness of an oriented fuzzy number is the entropy measure $e \in\left[\mathbb{R}_{0}^{+}\right]^{\mathbb{F}}$. This measure was also proposed by de Luca and Termini [60] and was then modified by Piasecki [61].

In [62], Kosko described the most widely known kind of entropy measure. Unfortunately, in $[44,45]$ it is shown that Kosko's entropy measure is not convenient for portfolio analysis. For this reason, in $[23,34]$ the tool for measuring the OFN indistinctness is proposed as the entropy measure $e \in\left[\mathbb{R}_{0}^{+}\right]^{\mathbb{K}}$, defined by the identity:

$e\left(\overleftrightarrow{\mathscr{L}}\left(a, b, c, d, L_{L}, R_{L}\right)\right)=\left|\int_{a}^{d} \min \left\{\mu_{L}\left(x \mid a, b, c, d, L_{L}, R_{L}\right), 1-\mu_{L}\left(x \mid a, b, c, d, L_{L}, R_{L}\right)\right\} d x\right|$.

This entropy measure is an extension of the entropy measure $e \in\left[\mathbb{R}_{0}^{+}\right]^{\mathbb{F}}$, introduced for FNs by Czogała, Gottwald, and Pedrycz in [63]. In decision analysis, we use the entropy measure as a measure of the indistinctness risk. Imprecision risk consists of both ambiguity and indistinctness risk combined.

For any $\operatorname{TrOFN} \stackrel{\leftrightarrow}{\operatorname{Tr}}(a, b, c, d)$, its energy and entropy are determined based on the following equations [34]:

$$
\begin{aligned}
& d(\overleftrightarrow{\operatorname{Tr}}(a, b, c, d))=\frac{1}{2} \cdot|d+c-b-a|, \\
& e(\overleftrightarrow{\operatorname{Tr}}(a, b, c, d))=\frac{1}{4} \cdot|d-c+b-a| .
\end{aligned}
$$

The results can be described in a form convenient for the analysis of an investment portfolio. An asset benefit index is the value of the relative profit of owned capital. This relative profit of the owned asset (for example return rate, discount factor) is a function that is dependent on the profit value and the asset value [34].

The benefit index for a two-assets portfolio is equal to the average of the portfolio component benefit indexes. This model is often used to analyze the effects of portfolio diversification. In our research, the portfolio component benefit indexes are imprecisely estimated. If portfolio component benefit indexes are described by trapezoidal oriented fuzzy numbers $\overleftrightarrow{\mathscr{K}}, \overleftrightarrow{\mathscr{L}} \in \mathbb{K}_{T r}$, then the portfolio benefit index is determined by the function $\omega:\left(\mathbb{K}_{T r}\right)^{2} \times[0,1] \rightarrow \mathbb{K}_{T r}$, given by this formula [34]:

$$
\omega(\overleftrightarrow{\mathscr{K}}, \overleftrightarrow{\mathscr{L}}, \lambda)=(\lambda \boxminus \overleftrightarrow{\mathscr{K}}) \boxplus((1-\lambda) \square \overleftrightarrow{\mathscr{L}})
$$

The method of determining the parameter $\lambda$ depends on the kind of considered relative benefits [45].

Theorem 2 [34].For any real number $\lambda \in[0,1]$, we have:

- $\quad$ for any pair $(\overleftrightarrow{\mathscr{K}}, \overleftrightarrow{\mathscr{L}}) \in\left(\mathbb{K}_{T r}^{-} \times \mathbb{K}_{T r}^{-}\right) \cup\left(\left(\mathbb{K}_{T r}^{+} \cup \mathbb{R}\right) \times\left(\mathbb{K}_{T r}^{+} \cup \mathbb{R}\right)\right)$

$$
\begin{aligned}
& d(\omega(\overleftrightarrow{\mathscr{K}}, \overleftrightarrow{\mathscr{L}}, \lambda))=\lambda \cdot d(\overleftrightarrow{\mathscr{K}})+(1-\lambda) \cdot d(\overleftrightarrow{\mathscr{L}}) \\
& e(\omega(\overleftrightarrow{\mathscr{K}}, \overleftrightarrow{\mathscr{L}}, \lambda))=\lambda \cdot e(\overleftrightarrow{\mathscr{K}})+(1-\lambda) \cdot e(\overleftrightarrow{\mathscr{L}}),
\end{aligned}
$$

- $\quad$ for any pair $(\overleftrightarrow{\mathscr{K}}, \overleftrightarrow{\mathscr{L}}) \in\left(\left(\mathbb{K}_{T r}^{+} \cup \mathbb{R}\right) \times \mathbb{K}_{T r}^{-}\right)$ 


$$
d(\varpi(\overleftrightarrow{\mathcal{K}}, \overleftrightarrow{\mathcal{L}}, \lambda)) \leq\left\{\begin{array}{l}
\lambda \cdot d(\overleftrightarrow{\mathcal{K}})-(1-\lambda) \cdot d(\operatorname{Core}(\overleftrightarrow{\mathcal{L}})), \varpi(\overleftrightarrow{\mathcal{K}}, \overleftrightarrow{\mathcal{L}}, \lambda) \in \mathbb{K}_{T r}^{+} \cup \mathbb{R} \\
(1-\lambda) \cdot d(\overleftrightarrow{\mathcal{L}})-\lambda \cdot d(\operatorname{Core}(\overleftrightarrow{\mathcal{K}})), \varpi(\overleftrightarrow{\mathcal{K}}, \overleftrightarrow{\mathcal{L}}, \lambda) \in \mathbb{K}_{T r}^{-} \cup \mathbb{R}
\end{array}\right.
$$

- $\quad$ for any pair $(\overleftrightarrow{\mathscr{K}}, \overleftrightarrow{\mathscr{L}}) \in\left(\left(\mathbb{K}_{T r}^{+} \cup \mathbb{R}\right) \times \mathbb{K}_{T r}^{-}\right) \cup\left(\mathbb{K}_{T r}^{-} \times\left(\mathbb{K}_{T r}^{+} \cup \mathbb{R}\right)\right)$

$$
e(\omega(\overleftrightarrow{\mathscr{K}}, \overleftrightarrow{\mathscr{L}}, \lambda)) \leq \min \{\lambda e(\overleftrightarrow{\mathscr{K}}),(1-\lambda) e(\overleftrightarrow{\mathscr{L}})\}
$$

\section{Oriented Fuzzy Present Value}

According to the uncertainty theory, any unknown future state of affairs is uncertain $[1,2]$. The uncertainty results from our lack of knowledge about the future state of affairs. We can model uncertainty with some probability. It is possible when we are able to indicate a specific time in the future, when the effect of a considered situation will already be known to the observer [3-9]. This was initially formulated by Kolmogorov [5,6] and is called "Kolmogorov's postulate".

We understand a security as an authorization to receive a future financial income payable to a certain maturity. The value of this income is understood as an anticipated future value of an asset. In our research, we can point to this particular time in the future at which the considered revenue will already be known to the observer. For this reason, the future value is not burdened by Knight's uncertainty [64]. Together with Kolmogorov's postulate, this allows us to conclude that the future value is a random variable.

In [12], a present value (PV) was redefined as a present equivalent of a payment available at a given time in the future. In our research, the estimation of the fuzzy present value is supplemented by a forecast of the closest changes in price. It was shown in [65] that the closest price changes can be forecasted using a prediction table. The use of oriented fuzzy numbers for a portfolio analysis is more useful than the use of fuzzy numbers, as discussed in detail in [34]. For this reason, an imprecise present value may be estimated using oriented fuzzy numbers [21,32]. PV, defined in this way, is called an oriented present value (O-PV). Any O-PV is described by a monotonic sequence $\left(V_{s}, V_{f}, \breve{P}, V_{l}, V_{e}\right)$ and then it is estimated by a trapezoidal oriented fuzzy number:

$$
\stackrel{\leftrightarrow}{P V}=\overleftrightarrow{\operatorname{Tr}}\left(V_{s}, V_{f}, V_{l}, V_{e}\right)
$$

where the monotonic sequence $\left(V_{s}, V_{f}, \check{P}, V_{l}, V_{e}\right)$ is determined in the following way:

- $\quad \check{P}$ is a quoted price.

- $\left[V_{s}, V_{e}\right] \subset \mathbb{R}^{+}$is an interval of all possible values of PV.

- $\left[V_{f}, V_{l}\right] \subset\left[V_{s}, V_{e}\right]$ is the interval of all prices that do not noticeably differ from the quoted price $\check{P}$.

If we anticipate a price increase, then PV is described by a positively oriented trapezoidal fuzzy number. If we anticipate a price decrease, then PV is described by a negatively oriented trapezoidal fuzzy number.

In our research, oriented present value is used for modelling imprecise evaluations of PV. The imprecision consists of ambiguity and indistinctness. We comprehend the ambiguity in such a way that neither value is unequivocally indicated. An indistinctness is comprehended in such a way that values equal to the present value and values different from the present value are not clearly distinguishable. We use the energy and entropy to measure the ambiguity and indistinctness of the O-PV given by $\stackrel{\leftrightarrow}{P V}=\stackrel{\leftrightarrow}{\operatorname{Tr}}\left(V_{s}, V_{f}, V_{l}, V_{e}\right)$. 
Let the fixed security Ŝ be given. Using Equations (18), (19) and (25) for fixed security $\hat{S}$, we get:

$$
\begin{aligned}
& d(\overleftrightarrow{P V}(\hat{S}))=\frac{1}{2} \cdot\left|V_{e}+V_{l}-V_{f}-V_{s}\right|, \\
& e(\stackrel{\leftrightarrow}{P V}(\hat{S}))=\frac{1}{4} \cdot\left|V_{e}-V_{l}+V_{f}-V_{s}\right| .
\end{aligned}
$$

Examples of trapezoidal O-PVs are the Japanese candle model [21] and the HeikinAshi candle model [24].

Example 1 [32]. We consider a portfolio of company stocks included in WIG20 quoted on the Warsaw Stock Exchange (WSE). Based on a session closing on the WSE on 28 January 2020, for each observed stock we evaluated its oriented present value assessed by trapezoidal oriented fuzzy number, describing its Japanese candle [21]. Table 1 contains these stocks' O-PVs. For each considered stock $\hat{S}$, we notice its quoted price $\breve{P}_{s}$ is the initial price on 29 January 2020.

\begin{tabular}{|c|c|c|c|c|}
\hline Company's Stock & Present Value $\overleftrightarrow{P V}_{s}$ & Quoted Price $\check{P}_{s}$ & $\begin{array}{c}\text { Energy } \\
\text { Measure }\end{array}$ & $\begin{array}{l}\text { Entropy } \\
\text { Measure }\end{array}$ \\
\hline ALR & $\overleftrightarrow{\operatorname{Tr}}(27.42 ; 27.30 ; 27.00 ; 26.84)$ & 27.00 & 0.44 & 0.07 \\
\hline CCC & $\overleftrightarrow{\operatorname{Tr}}(83.35 ; 88.00 ; 88.00 ; 89.65)$ & 88.00 & 3.15 & 1.575 \\
\hline CDR & $\overleftrightarrow{\operatorname{Tr}}(271.50 ; 271.50 ; 276.30 ; 276.30)$ & 277.00 & 4.8 & 0 \\
\hline CPS & $\overleftrightarrow{\operatorname{Tr}}(26.42 ; 26.60 ; 27.04 ; 27.34)$ & 27.20 & 0.68 & 0.12 \\
\hline DNP & $\overleftrightarrow{\operatorname{Tr}}(155.00 ; 155.00 ; 155.10 ; 157.30)$ & 155.30 & 1.2 & 0.55 \\
\hline JSW & $\stackrel{\leftrightarrow}{\operatorname{Tr}}(18.60 ; 19.36 ; 20.14 ; 20.14)$ & 20.32 & 1.16 & 0.19 \\
\hline KGH & $\overleftrightarrow{\operatorname{Tr}}(91.78 ; 93.60 ; 93.70 ; 94.90)$ & 94.24 & 1.61 & 0.755 \\
\hline LTS & $\overleftrightarrow{\operatorname{Tr}}(83.88 ; 83.40 ; 81.16 ; 80.26)$ & 81.44 & 2.93 & 0.345 \\
\hline LPP & $\overleftrightarrow{\operatorname{Tr}}(8205.00 ; 8380.00 ; 8395.00 ; 8460.00)$ & 8385.00 & 135 & 60 \\
\hline MBK & $\overleftrightarrow{\operatorname{Tr}}(367.00 ; 366.00 ; 359.80 ; 357.00)$ & 359.00 & 8.1 & 0.95 \\
\hline OPL & $\overleftrightarrow{\operatorname{Tr}}(7.01 ; 7.05 ; 7.20 ; 7.35)$ & 7.17 & 0.245 & 0.0475 \\
\hline PEO & $\overleftrightarrow{\operatorname{Tr}}(97.22 ; 97.70 ; 98.20 ; 98.66)$ & 98.20 & 0.97 & 0.235 \\
\hline PGE & $\stackrel{\leftrightarrow}{\operatorname{Tr}}(7.08 ; 7.15 ; 7.30 ; 7.40)$ & 7.30 & 0.235 & 0.0425 \\
\hline PGN & $\overleftrightarrow{\operatorname{Tr}}(3.91 ; 3.88 ; 3.86 ; 3.82)$ & 3.87 & 0.055 & 0.0175 \\
\hline PKN & $\stackrel{\leftrightarrow}{\operatorname{Tr}}(83.22 ; 83.00 ; 81.62 ; 81.18)$ & 81.90 & 1.71 & 0.165 \\
\hline PKO & $\stackrel{\leftrightarrow}{\operatorname{Tr}}(34.59 ; 34.68 ; 34.90 ; 35.26)$ & 34.93 & 0.445 & 0.1125 \\
\hline PLY & $\overleftrightarrow{\operatorname{Tr}}(35.82 ; 35.94 ; 36.76 ; 37.20)$ & 36.70 & 1.1 & 0.14 \\
\hline PZU & $\overleftrightarrow{\operatorname{Tr}}(40.72 ; 40.73 ; 40.89 ; 41.11)$ & 40.88 & 0.275 & 0.0575 \\
\hline SPL & $\overleftrightarrow{\operatorname{Tr}}(276.20 ; 278.00 ; 281.80 ; 283.80)$ & 287.00 & 5.7 & 0.95 \\
\hline TPE & $\stackrel{\leftrightarrow}{\operatorname{Tr}}(1.51 ; 1.53 ; 1.56 ; 1.56)$ & 1.56 & 0.04 & 0.005 \\
\hline
\end{tabular}

Table 1. Recorded values of the portfolio $\pi$ stocks.

We calculate the energy and entropy measure of the oriented present value using Equations (26) and (27), respectively.

It is noteworthy that CCC, CDR, CPS, DNP, JWS, KGH, LPP, OPL, PEO, PGE, PKO, PLY, PZU, SPL, and TPE are estimated by positively oriented trapezoidal fuzzy numbers. It means that they are evaluated by positively oriented present values. Hence, we anticipate that their quoted prices will increase. Conversely, ALR, LTS, MBK, PGN, and PKN are estimated by negatively oriented trapezoidal fuzzy numbers, which means that they are evaluated by negatively oriented present values; thus, we anticipate that their quoted prices will decrease. 


\section{Expected Discount Factor}

Let us assume that the time horizon $t>0$ of an investment is fixed. In this case, the considered asset is determined by two values:

- $\quad$ the anticipated future value $(\mathrm{FV}), V_{t}$.

- $\quad$ the assessed present value (PV), $V_{0}$.

The basic property of benefits from owning this asset is a simple return rate $r_{t}$, given by the formula:

$$
r_{t}=\frac{V_{t}-V_{0}}{V_{0}}=\frac{V_{t}}{V_{0}}-1 .
$$

According to Kolmogorov's postulate [5,6], FV is a random variable $\widetilde{V}_{t}: \Omega \longrightarrow \mathbb{R}^{+}$, where $\Omega$ is a set of elementary states $\omega$ of the financial market. In a classical approach to a return rate estimation, present value is identified with the observed quoted price $\breve{P}$. Thus, the return rate is a random variable determined by identity:

$$
r_{t}(\omega)=\frac{\widetilde{V}_{t}(\omega)-\check{P}}{\check{P}} .
$$

Uncertainty risk is a result of a lack of knowledge about the future state of affairs. In the practice of financial market analysis, the uncertainty risk is usually described by the probability distribution of return rate (29). This function may be given by a cumulative distribution function $F_{r}(\cdot \mid \bar{r}): \mathbb{R} \longrightarrow[0,1]$. We assume that the expected return rate $\bar{r}$ of this distribution exists. The expected discount factor (EDF) $\bar{v}$ must therefore also exist and is determined by the dependency:

$$
\bar{v}=(1+\bar{r})^{-1} .
$$

To simplify the reading of Equation (30) and further where Equation (30) is used, $\omega$ has been omitted.

Taking (28) and (29), we obtain the following formula describing the return rate:

$$
r_{t}=r_{t}\left(V_{0}, \omega\right)=\frac{\check{P} \cdot\left(1+r_{t}(\omega)\right)}{V_{0}}-1 .
$$

It implies that the expected return rate may be expressed in the following way:

$$
\mathscr{R}\left(V_{0}\right)=\int_{-\infty}^{+\infty} \frac{\check{P} \cdot(1+y)}{V_{0}}-1 d F_{r}(y \mid \bar{r})=\frac{\check{P} \cdot(1+\bar{r})}{V_{0}}-1 .
$$

In this way, we express the return rate as a decreasing unary operator $\mathscr{R}: \mathbb{R}^{+} \longrightarrow \mathbb{R}$ transforming PV. If PV is imprecisely estimated by TrOFN (25), then in line with (13), (14) and (15), the imprecise return rate is given as OFN:

$$
\begin{aligned}
\mathscr{R}(\hat{S})=\mathscr{R}(\overleftrightarrow{P V}(\hat{S})) & =\mathscr{R}\left(\overleftrightarrow{\operatorname{Tr}}\left(V_{s}, V_{f}, V_{l}, V_{e}\right)\right) \\
& =\overleftrightarrow{\mathscr{L}}\left(\frac{\overleftrightarrow{P} \cdot(1+\bar{r})}{V_{e}}-1, \frac{\check{P} \cdot(1+\bar{r})}{V_{l}}-1, \frac{\check{P} \cdot(1+\bar{r})}{V_{f}}-1, \frac{\check{P} \cdot(1+\bar{r})}{V_{s}}-1, S_{L}, E_{L}\right),
\end{aligned}
$$

where the reference functions are given by the formulas:

$$
\begin{aligned}
& \forall y \in\left[\frac{\check{P} \cdot(1+\bar{r})}{V_{s}}-1, \frac{\check{P} \cdot(1+\bar{r})}{V_{f}}-1\right] S_{L}(r)=\frac{\frac{\check{P} \cdot(1+\bar{r})}{1+r}-V_{s}}{V_{f}-V_{s}}, \\
& \forall y \in\left[\frac{\check{P} \cdot(1+\bar{r})}{V_{l}}-1, \frac{\check{P} \cdot(1+\bar{r})}{V_{e}}-1\right] E_{L}(r)=\frac{\frac{\check{P} \cdot(1+\bar{r})}{1+r}-V_{e}}{V_{l}-V_{e}} .
\end{aligned}
$$


It is noteworthy that the above reference functions are not linear. It implies that OFN (33) is not TrOFN. The use of the return rate does not allow us to bypass the complexity of the arithmetic operations of OFN.

In the next step, we consider the discount factor $v_{t}$, which is determined by the return rate $r_{t}$. From (28), we get:

$$
v_{t}=\left(1+r_{t}\right)^{-1} \text {. }
$$

Then, taking (36) and (29) together, we obtain the following random variable describing a discount factor:

$$
v_{t}=r_{t}\left(V_{0}, \omega\right)=\frac{V_{0}}{\check{P} \cdot\left(1+r_{t}(\omega)\right)} .
$$

This implies that the expected discount factor EDF may be expressed in the following way:

$$
\mathscr{V}\left(V_{0}\right)=\int_{-\infty}^{+\infty} \frac{V_{0}}{\check{P} \cdot(1+y)} d F_{r}(y \mid \bar{r})=\frac{V_{0}}{\check{P} \cdot(1+\bar{r})}=\left(\frac{\check{P} \cdot(1+\bar{r})}{V_{0}}\right)^{-1}=\frac{\bar{v}}{\breve{P}} \cdot V_{0} .
$$

Using this equation, we determine the imprecise EDF $\mathscr{V}: \mathbb{R}^{+} \rightarrow \mathbb{R}^{+}$as an increasing operator transforming PV. If PV is imprecisely estimated by TrOFN (25), then in line with (13)-(15), the imprecise EDF is given as TrOFN:

$$
\overleftrightarrow{\mathscr{V}}(\hat{S})=\overleftrightarrow{\mathscr{V}}(\overleftrightarrow{P V}(\hat{S}))=\overleftrightarrow{\mathscr{L}}\left(\frac{V_{s} \cdot \bar{v}}{\check{P}}, \frac{V_{f} \cdot \bar{v}}{\check{P}}, \frac{V_{l} \cdot \bar{v}}{\check{P}}, \frac{V_{e} \cdot \bar{v}}{\check{P}}, S_{L}, E_{L}\right)
$$

where the reference functions are given by the formulas:

$$
\begin{gathered}
\forall y \in\left[\frac{V_{s} \cdot \bar{v}}{\check{P}}, \frac{V_{f} \cdot \bar{v}}{\check{P}}\right] S_{L}(v)=\frac{\frac{v \cdot \breve{P}}{\bar{v}}-V_{s}}{V_{f}-V_{s}}=\frac{\check{P} \cdot v-V_{s} \cdot \bar{v}}{\bar{v} \cdot\left(V_{f}-V_{s}\right)}, \\
\forall y \in\left[\frac{V_{l} \cdot \bar{v}}{\check{P}}, \frac{e \cdot \bar{v}}{\check{P}}\right] E_{L}(v)=\frac{\frac{v \cdot \breve{P}}{\bar{v}}-V_{e}}{V_{l}-V_{e}}=\frac{\check{P} \cdot v-V_{e} \cdot \bar{v}}{\bar{v} \cdot\left(V_{l}-V_{e}\right)} .
\end{gathered}
$$

Note that both the above reference functions are linear. This implies that if an imprecise $\mathrm{PV}$ is given by TrOFN (25), then the imprecise EDF $\mathscr{V}$ is TrOFN, which is given by the formula:

$$
\overleftrightarrow{\mathscr{V}}(\hat{S})=\overleftrightarrow{\mathscr{V}}(\overleftrightarrow{P V}(\hat{S}))=\frac{\bar{v}}{\check{P}} \square \overleftrightarrow{P V}(\hat{S})
$$

In this case, it is clear that in financial analysis the imprecise EDF is more useful than the imprecise expected return rate. If we apply EDF, then the criterion of maximization of the expected return rate is replaced by the criterion of EDF minimalization.

Using (35), (18) and (19), the energy measure and the entropy measure of the expected discount factor EDF $\overleftrightarrow{\mathscr{V}}(\hat{S})$ are determined by the formulas:

$$
\begin{aligned}
& d(\overleftrightarrow{\mathscr{V}}(\hat{S}))=\left|\frac{\left(V_{e}+V_{l}-V_{f}-V_{s}\right) \cdot \overline{\mathcal{V}}}{2 \check{P}}\right|, \\
& e(\overleftrightarrow{\mathscr{V}}(\hat{S}))=\left|\frac{\left(V_{e}-V_{l}+V_{f}-V_{s}\right) \cdot \overline{\mathcal{V}}}{4 \check{P}}\right| .
\end{aligned}
$$

Example 2. All considerations in this paper are run for the quarterly period of the investment time $t=1$ quarter. We researched the stocks of the portfolio $\pi$ presented in Table 1 . For an average expected return rate $\bar{r}=0.10144$ from (30), we get an expected discount factor $\bar{v}=0.9079$. Using 
the one-year time series of quotations, for each considered stock Ŝsing (42), we calculate a quarterly expected discount factor O-EDF.

For example, for the O-EDF of ALR company stock, we get:

$$
\begin{aligned}
& \overleftrightarrow{\mathscr{V}}(A L R)=\overleftrightarrow{\mathscr{V}}(\overleftrightarrow{P V}(A L R))=\frac{\bar{v}}{\vec{P}} \sqcup \overleftrightarrow{P V}(A L R)=\frac{0.9079}{27} \square \overleftrightarrow{\operatorname{Tr}}(27.42 ; 27.30 ; 27.00 ; 26.84) \\
& =0.0336 \sqcup \overleftrightarrow{\operatorname{Tr}}(27.42 ; 27.30 ; 27.00 ; 26.84)=\overleftrightarrow{\operatorname{Tr}}(0.9220 ; 0.9180 ; 0.9079 ; 0.9025)
\end{aligned}
$$

We calculate the O-EDF in a similar way for other company stocks.

Additionally, using Equations (43) and (44), we determine, respectively, the energy and entropy measure of the O-EDF. All evaluations obtained in this way are presented in Table 2.

\begin{tabular}{|c|c|c|c|}
\hline Company's Stock & OEDF $\stackrel{\leftrightarrow}{V}_{s}$ & Energy Measure & Entropy Measure \\
\hline ALR & $\stackrel{\leftrightarrow}{\operatorname{Tr}}(0.9220 ; 0.9180 ; 0.9079 ; 0.9025)$ & 0.0148 & 0.0024 \\
\hline $\mathrm{CCC}$ & $\overleftrightarrow{\operatorname{Tr}}(0.8599 ; 0.9079 ; 0.9079 ; 0.9249)$ & 0.0325 & 0.0163 \\
\hline CDR & $\overleftrightarrow{\operatorname{Tr}}(0.8899 ; 0.8899 ; 0.9056 ; 0.9056)$ & 0.0157 & 0.0000 \\
\hline CPS & $\stackrel{\leftrightarrow}{\operatorname{Tr}}(0.8819 ; 0.8879 ; 0.9026 ; 0.9126)$ & 0.0227 & 0.0040 \\
\hline DNP & $\stackrel{\leftrightarrow}{\operatorname{Tr}}(0.9062 ; 0.9062 ; 0.9067 ; 0.9196)$ & 0.0070 & 0.0032 \\
\hline JSW & $\stackrel{\leftrightarrow}{\operatorname{Tr}}(0.8311 ; 0.8650 ; 0.8999 ; 0.8999)$ & 0.0518 & 0.0085 \\
\hline KGH & $\overleftrightarrow{\operatorname{Tr}}(0.8842 ; 0.9017 ; 0.9027 ; 0.9143)$ & 0.0155 & 0.0073 \\
\hline LTS & $\overleftrightarrow{\operatorname{Tr}}(0.9351 ; 0.9298 ; 0.9048 ; 0.8948)$ & 0.0327 & 0.0039 \\
\hline LPP & $\overleftrightarrow{\operatorname{Tr}}(0.8884 ; 0.9074 ; 0.9090 ; 0.9160)$ & 0.0146 & 0.0065 \\
\hline MBK & $\stackrel{\leftrightarrow}{\operatorname{Tr}}(0.9281 ; 0.9256 ; 0.9099 ; 0.9028)$ & 0.0205 & 0.0024 \\
\hline OPL & $\stackrel{\leftrightarrow}{\operatorname{Tr}}(0.8876 ; 0.8927 ; 0.9117 ; 0.9307)$ & 0.0310 & 0.0060 \\
\hline PEO & $\overleftrightarrow{\operatorname{Tr}}(0.8988 ; 0.9033 ; 0.9079 ; 0.9122)$ & 0.0090 & 0.0022 \\
\hline PGE & $\overleftrightarrow{\operatorname{Tr}}(0.8805 ; 0.8892 ; 0.9079 ; 0.9203)$ & 0.0292 & 0.0053 \\
\hline PGN & $\stackrel{\leftrightarrow}{\operatorname{Tr}}(0.9173 ; 0.9103 ; 0.9056 ; 0.8962)$ & 0.0129 & 0.0041 \\
\hline PKN & $\overleftrightarrow{\operatorname{Tr}}(0.9225 ; 0.9201 ; 0.9048 ; 0.8999)$ & 0.0190 & 0.0018 \\
\hline PKO & $\stackrel{\leftrightarrow}{\operatorname{Tr}}(0.8991 ; 0.9014 ; 0.9071 ; 0.9165)$ & 0.0116 & 0.0029 \\
\hline PLY & $\stackrel{\leftrightarrow}{\operatorname{Tr}}(0.8861 ; 0.8891 ; 0.9094 ; 0.9203)$ & 0.0272 & 0.0035 \\
\hline PZU & $\stackrel{\leftrightarrow}{\operatorname{Tr}}(0.9044 ; 09046 ; 0.9081 ; 0.9130)$ & 0.0061 & 0.0013 \\
\hline SPL & $\overleftrightarrow{\operatorname{Tr}}(0.8737 ; 0.8794 ; 0.8915 ; 0.8978)$ & 0.0180 & 0.0030 \\
\hline TPE & $\overleftrightarrow{\operatorname{Tr}}(0.8788 ; 0.8904 ; 0.9079 ; 0.9079)$ & 0.0233 & 0.0029 \\
\hline
\end{tabular}

Table 2. O-EDF of portfolio $\pi$ components and their energy and entropy measures.

Note that the O-EDF of a security described in this way is a TrOFN with an identical orientation to the O-PV used for its estimation.

\section{Expected Discount Factors for Portfolio}

By a financial portfolio, we mean an arbitrary, finite set of assets. Any asset is treated as a fixed security in a long position. On the other hand, any portfolio is also a security. Let us consider the case of a multi-asset portfolio $\pi^{*}$, built of assets $Y_{i}$. We describe this portfolio as the set $\pi^{*}=\left\{Y_{i}: i=1,2, \ldots, n\right\}$. Any asset $Y_{i}$ is determined by a block of $n_{i}$ of stocks $\hat{S}_{i}$ quoted at the price $\breve{P}_{i} \in \mathbb{R}^{+}$. Any security is characterized by its imprecise PV evaluated by TrOFN:

$$
\overleftrightarrow{\operatorname{Tr}}\left(V_{s}^{(i)}, V_{f}^{(i)}, V_{l}^{(i)}, V_{e}^{(i)}\right)
$$

This means that:

$$
\overleftrightarrow{P V}\left(Y_{i}\right)=n_{i} \boxminus \stackrel{\leftrightarrow}{P V}\left(\hat{S}_{i}\right)
$$

where the quoted value is equal to:

$$
M_{i}=n_{i} \cdot \check{P}_{i}
$$


and by its $\operatorname{EDF} \bar{v}_{i}$ determined by (30). Taking into account all of the above, we evaluate any asset $Y_{i}$ by its imprecise EDF

$$
\overleftrightarrow{\mathscr{V}}\left(Y_{i}\right)=\overleftrightarrow{\mathscr{V}}\left(\hat{S}_{i}\right)=\overleftrightarrow{\operatorname{Tr}}\left(D_{s}^{(i)}, D_{f}^{(i)}, D_{l}^{(i)}, D_{e}^{(i)}\right)
$$

Example 3. We construct a portfolio $\pi$ consisting of the stocks presented in Table 1. Table 3 contains information on the number of stocks of a given company in the portfolio, the value of the current block of these stocks, and their price. For example, for ALR we get, from (46):

$$
\begin{aligned}
& \stackrel{\leftrightarrow}{P V}\left(Y_{1}\right)=170 \boxminus \overleftrightarrow{P V}(A L R)=170 \bullet \overleftrightarrow{\operatorname{Tr}}(27.42 ; 27.30 ; 27.00 ; 26.84) \\
& =\overleftrightarrow{\operatorname{Tr}}(4661.40 ; 4641.00 ; 4590.00 ; 4562.80)
\end{aligned}
$$

\begin{tabular}{|c|c|c|c|c|}
\hline Assets & Company's Stock & Number of Stocks in Block & Present Value $\overleftrightarrow{P V}_{s}$ & Price $M_{i}$ \\
\hline$Y_{1}$ & ALR & 170 & $\overleftrightarrow{\operatorname{Tr}}(4661.40 ; 4641.00 ; 4590.00 ; 4562.80)$ & 4590.00 \\
\hline$Y_{2}$ & $\mathrm{CCC}$ & 10 & $\stackrel{\leftrightarrow}{\operatorname{Tr}}(833.50 ; 880.00 ; 880.00 ; 896.50)$ & 880.00 \\
\hline$Y_{3}$ & CDR & 17 & $\overleftrightarrow{\operatorname{Tr}}(4615.50 ; 4615.50 ; 4697.10 ; 4697.10)$ & 4709.00 \\
\hline$Y_{4}$ & CPS & 50 & $\overleftrightarrow{\operatorname{Tr}}(1321.00 ; 1330.00 ; 1352.00 ; 1367.00)$ & 1360.00 \\
\hline$Y_{5}$ & DNP & 5 & $\overleftrightarrow{\operatorname{Tr}}(775.00 ; 775.00 ; 775.50 ; 786.50)$ & 776.50 \\
\hline$Y_{6}$ & JSW & 200 & $\overleftrightarrow{\operatorname{Tr}}(3720.00 ; 3872.00 ; 4028.00 ; 4028.00)$ & 4064.00 \\
\hline$Y_{7}$ & $\mathrm{KGH}$ & 8 & $\overleftrightarrow{\operatorname{Tr}}(734.24 ; 748.80 ; 749.60 ; 759.20)$ & 769.92 \\
\hline$Y_{8}$ & LTS & 50 & $\overleftrightarrow{\operatorname{Tr}}(4194.00 ; 4170.00 ; 4058.00 ; 4013.00)$ & 4072.00 \\
\hline$Y_{9}$ & LPP & 1 & $\overleftrightarrow{\operatorname{Tr}}(8205.00 ; 8380.00 ; 8395.00 ; 8460.00)$ & 8385.00 \\
\hline$Y_{10}$ & MBK & 25 & $\overleftrightarrow{\operatorname{Tr}}(9175.00 ; 9150.00 ; 8995.00 ; 8925.00)$ & 8975.00 \\
\hline$Y_{11}$ & OPL & 100 & $\stackrel{\leftrightarrow}{\operatorname{Tr}}(701.00 ; 705.00 ; 720.00 ; 735.00)$ & 717.00 \\
\hline$Y_{12}$ & PEO & 10 & $\overleftrightarrow{\operatorname{Tr}}(972.20 ; 977.00 ; 982.00 ; 986.60)$ & 982.00 \\
\hline$Y_{13}$ & PGE & 100 & $\overleftrightarrow{\operatorname{Tr}}(708.00 ; 715.00 ; 730.00 ; 740.00)$ & 730.00 \\
\hline$Y_{14}$ & PGN & 1200 & $\overleftrightarrow{\operatorname{Tr}}(4692.00 ; 4656.00 ; 4632.00 ; 4584.00)$ & 4644.00 \\
\hline$Y_{15}$ & PKN & 50 & $\overleftrightarrow{\operatorname{Tr}}(4161.00 ; 4150.00 ; 4081.00 ; 4059.00)$ & 4095.00 \\
\hline$Y_{16}$ & PKO & 30 & $\overleftrightarrow{\operatorname{Tr}}(1037.70 ; 1040.40 ; 1047.00 ; 1057.80)$ & 1047.90 \\
\hline$Y_{17}$ & PLY & 60 & $\overleftrightarrow{\operatorname{Tr}}(2149.20 ; 2156.40 ; 2205.60 ; 2232.00)$ & 2202.00 \\
\hline$Y_{18}$ & PZU & 25 & $\overleftrightarrow{\operatorname{Tr}}(1018.00 ; 1018.25 ; 1022.25 ; 1027.75)$ & 1022.00 \\
\hline$Y_{19}$ & SPL & 10 & $\overleftrightarrow{\operatorname{Tr}}(2762.00 ; 2780.00 ; 2818.00 ; 2838.00)$ & 2870.00 \\
\hline$Y_{20}$ & $\mathrm{TPE}$ & 200 & $\stackrel{\leftrightarrow}{\operatorname{Tr}}(302.00 ; 306.00 ; 312.00 ; 312.00)$ & 312.00 \\
\hline
\end{tabular}

and from (47), we get: $M_{1}=170 \cdot 27.00=4590.00$. We do the same with the other assets.

Table 3. Recorded values of the portfolio $\pi$ stocks.

The present value of the portfolio is the sum of the present values of its components. The components' PVs are estimated by trapezoidal oriented fuzzy numbers. The addition of TrOFN is not associative. Additionally, multiple addition depends on the order of the components. For this reason, a portfolio's present value, given as the sum of its components' PVs, is not clearly defined. Therefore, calculating the portfolio PV, we order the portfolio components. We use a method of ordering the assets proposed and justified in [22]. At the outset, we distinguish the portfolio of rising assets $\pi^{+}=\left\{Y_{i} \in \pi^{*}: \stackrel{\leftrightarrow}{P} V_{i} \in \mathbb{K}_{T r}^{+}\right\}$and the portfolio of falling assets $\pi^{-}=\pi^{*} \backslash \pi^{+}$. Then, using (29), we calculate the PV of portfolio 
$\pi^{+}$, denoted by the symbol $\stackrel{\leftrightarrow}{P V}^{+}$, and the PV of portfolio $\pi^{-}$, denoted by the symbol $\stackrel{\leftrightarrow}{P V}^{+}$. Thus, we get:

$$
\begin{aligned}
& \overleftrightarrow{P V}^{+}=\overleftrightarrow{\operatorname{Tr}}\left(V_{s}^{(+)}, V_{f}^{(+)}, V_{l}^{(+)}, V_{e}^{(+)}\right)=\overleftrightarrow{\operatorname{Tr}}\left(\sum_{Y_{i} \in \pi^{+}} V_{s}^{(i)}, \sum_{Y_{i} \in \pi^{+}} V_{f}^{(i)}, \sum_{Y_{i} \in \pi^{+}} V_{l}^{(i)}, \sum_{Y_{i} \in \pi^{+}} V_{e}^{(i)}\right) \\
& \overleftrightarrow{P V}^{-}=\overleftrightarrow{\operatorname{Tr}}\left(V_{s}^{(-)}, V_{f}^{(-)}, V_{l}^{(-)}, V_{e}^{(-)}\right)=\overleftrightarrow{\operatorname{Tr}}\left(\sum_{Y_{i} \in \pi^{-}} V_{s}^{(i)}, \sum_{Y_{i} \in \pi^{-}} V_{f}^{(i)}, \sum_{Y_{i} \in \pi^{-}} V_{l}^{(i)}, \sum_{Y_{i} \in \pi^{-}} V_{e}^{(i)}\right) .
\end{aligned}
$$

Finally, we calculate the PV of portfolio $\pi^{*}$, denoted by the symbol $\stackrel{\leftrightarrow}{P V}^{*}$. We then get:

$$
\overleftrightarrow{P V}^{*}=\overleftrightarrow{P V}^{+} \boxplus \overleftrightarrow{P V}^{-}=\overleftrightarrow{\operatorname{Tr}}\left(V_{s}^{(+)}, V_{f}^{(+)}, V_{l}^{(+)}, V_{e}^{(+)}\right) \boxplus \overleftrightarrow{\operatorname{Tr}}\left(V_{s}^{(-)}, V_{f}^{(-)}, V_{l}^{(-)}, V_{e}^{(-)}\right)=\overleftrightarrow{\operatorname{Tr}}\left(V_{s}^{(*)}, V_{f}^{(*)}, V_{l}^{(*)}, V_{e}^{(*)}\right)
$$

Example 4. For the portfolio determined in Example 3, we have the portfolio of rising assets:

$$
\pi^{+}=\left\{Y_{2}, Y_{3}, Y_{4}, Y_{5}, Y_{6}, Y_{7}, Y_{9}, Y_{11}, Y_{12}, Y_{13}, Y_{16}, Y_{17}, Y_{18}, Y_{19}, Y_{20}\right\}
$$

and the portfolio of falling assets:

$$
\pi^{-}=\left\{Y_{1}, Y_{8}, Y_{10}, Y_{14}, Y_{15}\right\}
$$

Using (49), (50) and (51), we get the O-PV of portfolios $\pi^{+}, \pi^{-}$, and $\pi^{*}$, respectively:

$$
\begin{aligned}
& \stackrel{\leftrightarrow}{P V}^{+}=\overleftrightarrow{\operatorname{Tr}}(29854.34,30299.35,30714.05,30923.45) \\
& \overleftrightarrow{P V}^{-}=\overleftrightarrow{\operatorname{Tr}}(26883.40,26767.00,26356.00,26143.80) \\
& \stackrel{\leftrightarrow}{P V}^{*}=\stackrel{\leftrightarrow}{P V}^{+} \boxplus \overleftrightarrow{P V}^{-}=\overleftrightarrow{\operatorname{Tr}}(56737.74,57066.35,57070.05,57070.05)
\end{aligned}
$$

Now we can start calculating the EDFs of the considered portfolios. The values of portfolios $\pi^{+}, \pi^{-}$, and $\pi^{*}$ are calculated in the following way, respectively:

$$
M^{+}=\sum_{Y_{i} \in \pi^{+}} M_{i}, M^{-}=\sum_{Y_{i} \in \pi^{-}} M_{i}, M^{*}=M^{+}+M^{-} .
$$

The share $q_{i}^{+}$of the asset $Y_{i} \in \pi^{+}$in the portfolio $\pi^{+}$and the share $q_{i}^{-}$of the asset $Y_{i} \in \pi^{-}$in the portfolio $\pi^{-}$are given by the formulas:

$$
q_{i}^{+}=\frac{M_{i}}{M^{+}}, q_{i}^{-}=\frac{M_{i}}{M^{-}} .
$$

The share $q^{+}$of portfolio $\pi^{+}$in the portfolio $\pi^{*}$ and the share $q^{-}$of portfolio $\pi^{-}$in the portfolio $\pi^{*}$ are given by the formulas:

$$
q^{+}=\frac{M^{+}}{M^{*}}, q^{-}=\frac{M^{-}}{M^{*}} .
$$

The EDF $\bar{v}^{+}$of portfolio $\pi^{+}$, the EDF $\bar{v}^{-}$of portfolio $\pi^{-}$, and the EDF $\bar{v}^{*}$ of portfolio $\pi^{*}$ are calculated as follows:

$$
\bar{v}^{+}=\left(\sum_{\Upsilon_{i} \in \pi^{+}} \frac{q_{i}^{+}}{\bar{v}_{i}}\right)^{-1}, \bar{v}^{-}=\left(\sum_{\Upsilon_{i} \in \pi^{-}} \frac{q_{i}^{-}}{\bar{v}_{i}}\right)^{-1}, \bar{v}^{*}=\left(\frac{q^{+}}{\bar{v}^{+}}+\frac{q^{-}}{\bar{v}^{-}}\right)^{-1} .
$$


Due to the results obtained in $[21,34,44]$ and (12), we find that:

- the imprecise EDF $\overleftrightarrow{\mathscr{V}}^{+}$of portfolio $\pi^{+}$is given by the formula:

$$
\overleftrightarrow{\mathscr{V}}^{+}=\overleftrightarrow{\operatorname{Tr}}\left(D_{s}^{(+)}, D_{f}^{(+)}, D_{l}^{(+)}, D_{e}^{(+)}\right)=\bar{v}^{+} \square\left(\bigsqcup_{Y_{i} \in \pi^{+}}\left(\frac{q_{i}^{+}}{\bar{v}_{i}} \square \overleftrightarrow{\mathscr{V}}\left(Y_{i}\right)\right)\right)=\overline{T r}\left(\sum_{Y_{i} \in \pi^{+}} \frac{\bar{v}^{+} \cdot q_{i}^{(+)}}{\bar{v}_{i}} \cdot D_{s}^{(i)}, \sum_{Y_{i} \in \pi^{+}} \frac{\bar{v}^{+} \cdot q_{i}^{(+)}}{\bar{v}_{i}} \cdot D_{f}^{(i)}, \sum_{Y_{i} \in \pi^{+}} \frac{\bar{v}^{+} \cdot q_{i}^{(+)}}{\bar{v}_{i}} \cdot D_{l}^{(i)}, \sum_{Y_{i} \in \pi^{+}} \frac{\bar{v}^{+} \cdot q_{i}^{(+)}}{\bar{v}_{i}} \cdot D_{e}^{(i)}\right),
$$

- the imprecise EDF $\overleftrightarrow{\mathscr{V}}^{-}$of portfolio $\pi^{-}$is given by the formula:

$\overleftrightarrow{\mathscr{V}}^{-}=\overleftrightarrow{\operatorname{Tr}}\left(D_{s}^{(-)}, D_{f}^{(-)}, D_{l}^{(-)}, D_{e}^{(-)}\right)=\bar{v}^{-} \square\left(-\left(\frac{q_{i}^{-}}{\bar{v}_{i}} \square \overleftrightarrow{\mathscr{V}}\left(Y_{i}\right)\right)\right)=\overline{T r}\left(\sum_{Y_{i} \in \pi^{-}} \frac{\bar{v}^{-} \cdot q_{i}^{(-)}}{\bar{v}_{i}} \cdot D_{s}^{(i)}, \sum_{Y_{i} \in \pi^{-}} \frac{\bar{v}^{-} \cdot q_{i}^{(-)}}{\bar{v}_{i}} \cdot D_{f}^{(i)}, \sum_{Y_{i} \in \pi^{-}} \frac{\bar{v}^{-} \cdot q_{i}^{(-)}}{\bar{v}_{i}} \cdot D_{l}^{(i)}, \sum_{Y_{i} \in \pi^{-}} \frac{\bar{v}^{-} \cdot q_{i}^{(-)}}{\bar{v}_{i}} \cdot D_{e}^{(i)}\right)$,

- $\quad$ the imprecise EDF $\overleftrightarrow{\mathscr{V}}^{*}$ of portfolio $\pi^{*}$ is given by the formula:

$$
\overleftrightarrow{\mathscr{V}}^{*}=\overleftrightarrow{\operatorname{Tr}}\left(D_{s}^{(*)}, D_{f}^{(*)}, D_{l}^{(*)}, D_{e}^{(*)}\right)=\left(\frac{\bar{v}^{*} \cdot q^{+}}{\bar{v}^{+}} \boxminus \overleftrightarrow{\mathscr{V}}^{+}\right) \boxplus\left(\frac{\bar{v}^{*} \cdot q^{-}}{\bar{v}^{-}} \boxminus \overleftrightarrow{\mathscr{V}}^{-}\right)
$$

Then, using (56), (21) and (22), and mathematical induction, we find that the energy measure and the entropy measure of EDF $\overleftrightarrow{\mathscr{V}}^{+}$are determined by the formulas:

$$
\begin{aligned}
& d\left(\overleftrightarrow{\mathscr{V}}^{+}\right)=\sum_{Y_{i} \in \pi^{+}} \frac{\bar{v}^{+} \cdot q_{i}^{(+)}}{\bar{v}_{i}} \cdot d\left(\overleftrightarrow{\mathscr{V}}\left(Y_{i}\right)\right), \\
& e\left(\overleftrightarrow{\mathscr{V}}^{+}\right)=\sum_{Y_{i} \in \pi^{+}} \frac{\bar{v}^{+} \cdot q_{i}^{(+)}}{\bar{v}_{i}} \cdot e\left(\overleftrightarrow{\mathscr{V}}\left(Y_{i}\right)\right) .
\end{aligned}
$$

Similarly, using (57), (21) and (22), and mathematical induction, we find that the energy measure and the entropy measure of EDF $\overleftrightarrow{\mathscr{V}}^{-}$are determined by the formulas:

$$
\begin{aligned}
& d\left(\overleftrightarrow{\mathscr{V}}^{-}\right)=\sum_{Y_{i} \in \pi^{-}} \frac{\bar{v}^{-} \cdot q_{i}^{(-)}}{\bar{v}_{i}} \cdot d\left(\overleftrightarrow{\mathscr{V}}\left(Y_{i}\right)\right), \\
& e\left(\overleftrightarrow{\mathscr{V}}^{-}\right)=\sum_{Y_{i} \in \pi^{-}} \frac{\bar{v}^{-} \cdot q_{i}^{(-)}}{\bar{v}_{i}} \cdot e\left(\overleftrightarrow{\mathscr{V}}\left(Y_{i}\right)\right) .
\end{aligned}
$$

From (58), (23) and (24), the energy measure and entropy measure of EDF $\overleftrightarrow{\mathscr{V}}^{*}$ of portfolio $\pi^{*}$ meet the following conditions:

$$
\begin{array}{r}
d\left(\overleftrightarrow{\mathcal{V}}^{*}\right) \leq\left\{\begin{array}{l}
\frac{\bar{v}^{*} \cdot q^{+}}{\bar{v}^{+}} \cdot d\left(\overleftrightarrow{\mathcal{V}}^{+}\right)-\frac{\bar{v}^{*} \cdot q^{-}}{\bar{v}^{-}} \cdot d\left(\operatorname{Cor} e\left(\overleftrightarrow{\mathcal{V}}^{-}\right)\right), \overleftrightarrow{\mathcal{V}}^{*} \in \mathbb{K}_{T r}^{+} \cup \mathbb{R}, \\
\frac{\bar{v}^{*} \cdot q^{-}}{\bar{v}^{-}} \cdot d\left(\overleftrightarrow{\mathcal{V}}^{-}\right)-\frac{\bar{v}^{*} \cdot q^{+}}{\bar{v}^{+}} \cdot d\left(\operatorname{Cor} e\left(\overleftrightarrow{\mathcal{V}}^{+}\right)\right), \overleftrightarrow{\mathcal{V}}^{*} \in \mathbb{K}_{T r}^{-} \cup \mathbb{R}, \\
e\left(\overleftrightarrow{\mathscr{V}}^{*}\right) \leq \min \left\{\frac{\bar{v}^{*} \cdot q^{+}}{\bar{v}^{+}} \cdot e\left(\overleftrightarrow{\mathscr{V}}^{+}\right), \frac{\bar{v}^{*} \cdot q^{-}}{\bar{v}^{-}} \cdot e(\overleftrightarrow{\mathscr{V}})\right\}
\end{array}\right.
\end{array}
$$

Example 5. For the portfolio used in Example 3, we use (52) to calculate the quoted values of portfolios $\pi^{+}, \pi^{-}$, and $\pi^{*}$ and we get $M^{+}=30,827.32, M^{-}=26,376.00$, and $M^{*}=57,203.32$. Table 4 contains information on the share $q_{i}^{+}$of the asset $Y_{i} \in \pi^{+}$in the portfolio $\pi^{+}$and the share $q_{i}^{-}$of the asset $Y_{i} \in \pi^{-}$in the portfolio $\pi^{-}$. We obtained these values using (53). 
Table 4. The share $q_{i}^{+}$of the asset $Y_{i} \in \pi^{+}$in the portfolio $\pi^{+}$and the share $q_{i}^{-}$of the asset $Y_{i} \in \pi^{-}$ in the portfolio $\pi^{-}$.

\begin{tabular}{cc}
\hline Company & Share of the Asset in the Portfolio \\
\hline ALR & 0.1740 \\
CCC & 0.0286 \\
CDR & 0.1528 \\
CPS & 0.0441 \\
DNP & 0.0252 \\
JSW & 0.1318 \\
KGH & 0.0250 \\
LTS & 0.1544 \\
LPP & 0.2720 \\
MBK & 0.3403 \\
OPL & 0.0233 \\
PEO & 0.0319 \\
PGE & 0.0237 \\
PGN & 0.1761 \\
PKN & 0.1553 \\
PKO & 0.0340 \\
PLY & 0.0714 \\
PZU & 0.0332 \\
SPL & 0.0931 \\
TPE & 0.0101 \\
\hline
\end{tabular}

From (54), we calculate the share $q^{+}=0.5389$ of portfolio $\pi^{+}$in the portfolio $\pi^{*}$ and the share $q^{-}=0.4611$ of portfolio $\pi^{-}$in the portfolio $\pi^{*}$. Using (55), we obtain the $\mathrm{EDF} \bar{v}^{+}=0.9077$ of portfolio $\pi^{+}$, the EDF $\bar{v}^{-}=0.9078$ of portfolio $\pi^{-}$, and the EDF $\bar{v}^{*}=0.90775$ of portfolio $\pi^{*}$. Utilising (56), (57) and (58), we calculate the imprecise expected discount factors of portfolios $\pi^{+}, \pi^{-}$and $\pi^{*}$, respectively:

$$
\begin{aligned}
& \overleftrightarrow{\mathscr{V}}^{+}=\overleftrightarrow{\operatorname{Tr}}(0.8797,0.8927,0.9050,0.9112), \\
& \overleftrightarrow{\mathscr{V}}^{-}=\overleftrightarrow{\operatorname{Tr}}(0.9253,0.9214,0.9072,0.8999), \\
& \overleftrightarrow{\mathscr{V}}^{*}=\overleftrightarrow{\operatorname{Tr}}(0.9008,0.9060,0.9060,0.9060),
\end{aligned}
$$

The energy measure of EDF $\overleftrightarrow{\mathscr{V}}^{*}$ of portfolio $\pi^{*}$ equals $d\left(\overleftrightarrow{\mathscr{V}}^{*}\right)=0.0027$. On the other hand, using (59) and (61) we get $d\left(\overleftrightarrow{\mathscr{V}}^{+}\right)=0.0218$ and $d\left(\overleftrightarrow{\mathscr{V}}^{-}\right)=0.0198$ as well as $d\left(\operatorname{Core}\left(\overleftrightarrow{\mathcal{V}}^{-}\right)\right)=0.0142$, which is $d\left(\overleftrightarrow{\mathscr{V}}^{*}\right) \leq 0.0053$, which means that the condition (63) is satisfied. Additionally, we get $d\left(\overleftrightarrow{\mathscr{V}}^{*}\right)=0.0027 \leq 0.0053 \leq \min \left\{d\left(\overleftrightarrow{\mathscr{V}}^{+}\right), d\left(\overleftrightarrow{\mathscr{V}}^{-}\right)\right\}$. Similarly, the entropy measure of EDF $\overleftrightarrow{\mathscr{V}}^{*}$ of portfolio $\pi^{*}$ is $e\left(\overleftrightarrow{\mathscr{V}}^{*}\right)=0.0013$. On the other hand, using (60) and (62) we get $e\left(\overleftrightarrow{\mathscr{V}}^{+}\right)=0.0048$ and $e\left(\overleftrightarrow{\mathscr{V}}^{*}\right)=0.0028$, meaning that $e\left(\overleftrightarrow{\mathscr{V}}^{*}\right)=0.0013 \leq \min \left\{e\left(\overleftrightarrow{\mathscr{V}}^{+}\right), e\left(\overleftrightarrow{\mathscr{V}}^{-}\right)\right\}$, which means that the condition (64) is also satisfied. It is worth stressing that in [25] it was indicated that linear portfolio analysis is not possible for the considered portfolio $\pi^{*}$.

The above results allow us to conclude that portfolio diversification reduces uncertainty risk and imprecision risk. Additionally, it is worth noting that different orientations of index profits significantly affect ambiguity and indistinctness of portfolio profit index. 
Note that if index profits are described by TrOFNs with different orientations then portfolio diversification significantly reduces the ambiguity of the portfolio profit index and if the index profits are described by TrOFNs with the same orientations, then portfolio diversification only averages the ambiguity of the portfolio profit index. Similarly, if index profits are described by TrOFNs with different orientations, then portfolio diversification significantly reduces the indistinctness of the portfolio profit index and if index profits are described by TrOFNs with the same orientations, then portfolio diversification only averages the indistinctness of the portfolio profit index [23].

In order to make it easier for the reader to use the presented method, we present it in a shortened form using a simple algorithm:

We consider a portfolio of company stocks.

STEP 1. Based on a session closing on, for example, the Warsaw Stock Exchange, on a fixed day, for each observed stock we assess its O-PV equal to TrOFN, describing its Japanese candle and we calculate the energy and entropy measure of the oriented present value.

STEP 2. For each considered stock $\hat{\mathrm{S}}$, we note its quoted price $\check{\mathrm{P}}_{\mathrm{S}}$ as the initial price on the next day.

STEP 3. For each considered stocks $\hat{S}$, we calculate a quarterly expected discount factor O-EDF.

STEP 4 . We construct a portfolio $\pi$ consisting of the stocks from STEP 1 . We determine the number of stocks of a given company in the portfolio and calculate the value of the current block of these stocks and their price $M_{i}$.

STEP 5. For the portfolio from STEP 4, we describe the portfolio of the rising assets $\pi^{+}$and the portfolio of the falling assets $\pi^{-}$. We calculate the O-PV of portfolio $\pi^{+}, \pi^{-}$, and $\pi^{*}$, i.e., $\overleftrightarrow{P V}^{+}, \overleftrightarrow{P V}^{-}$, and $\overleftrightarrow{P V}^{*}=\overleftrightarrow{P V}^{+} \boxplus \overleftrightarrow{P V}^{-}$.

STEP 6. We calculate the values of the portfolios $\pi^{+}, \pi^{-}$, and $\pi^{*}$, i.e., $M^{+}=\sum Y_{i} \in \pi^{+} M_{i}$, $M^{-}=\sum_{Y_{i} \in \pi^{-}} M_{i}$ and $M^{*}=M^{+}+M^{-}$, the share $q_{i}^{+}=\frac{M_{i}}{M^{+}}$of portfolio $\pi^{+}$in portfolio $\pi^{*}$ and the share $q_{i}^{-}=\frac{M_{i}}{M^{-}}$of portfolio $\pi^{-}$in portfolio $\pi^{*}$, the share $q^{+}=\frac{M^{+}}{M^{*}}$ of portfolio $\pi^{+}$in portfolio $\pi^{*}$ and the share $q^{-}=\frac{M^{-}}{M^{*}}$ of portfolio $\pi^{-}$in portfolio $\pi^{*}$.

STEP 7. We calculate the $\operatorname{EDF} \bar{v}^{+}=\left(\sum Y_{i} \in \pi^{+} \frac{q_{i}^{+}}{\bar{v}_{i}}\right)^{-1}$ of portfolio $\pi^{+}$, the $\operatorname{EDF} \bar{v}^{-}=$ $\left(\sum Y_{i} \in \pi^{-} \frac{q_{i}^{-}}{\bar{v}_{i}}\right)^{-1}$ of portfolio $\pi^{-}$, and the EDF $\bar{v}^{*}=$ of portfolio $\pi^{*}$.

STEP 8. We calculate the imprecise EDF $\overleftrightarrow{\mathscr{V}}^{+}=\bar{v}^{+} \bullet\left(+_{Y_{i} \in \pi^{+}}\left(\frac{q_{i}^{+}}{\overline{\bar{v}}_{i}} \square \overleftrightarrow{\mathscr{V}}\left(Y_{i}\right)\right)\right)$ of portfolio $\pi^{+}$, the imprecise EDF $\overleftrightarrow{\mathscr{V}}^{-}=\bar{v}^{-} \triangleright\left(-\left(\frac{q_{i}^{-}}{\overline{\bar{v}}_{i}} \sqcup \overleftrightarrow{\mathscr{V}}\left(Y_{i}\right)\right)\right)$ of portfolio $\pi^{-}$, and the imprecise EDF $\overleftrightarrow{\mathscr{V}}^{*}=\left(\frac{\bar{v}^{*} \cdot q^{+}}{\bar{v}^{+}} \square \overleftrightarrow{\mathscr{V}}^{+}\right) \boxplus\left(\frac{\bar{v}^{*} \cdot q^{-}}{\bar{v}^{-}} \square \overleftrightarrow{\mathscr{V}}^{*}\right)$ of portfolio $\pi^{*}$ and we calculate the energy and entropy measures of these imprecise EDFs.

\section{Conclusions}

Statements describing particular definitions and facts influence the state of knowledge. Linguistic variables (variables with values that are actually words or sentences of natural language) are used to formulate statements. After Knight, imprecise information is identified with imperfect information. In the imprecision of information, we often distinguish ambiguity and indistinctness $[10,66]$. The ambiguity of information can be defined as a lack of unequivocal distinction of recommended options among many considered alternatives. Indistinctness of information is defined as a lack of unequivocal distinction between an information and its contradiction. Such a formal model of imprecision is a fuzzy set membership function. In our paper, imprecise information about a given financial instrument 
is presented with the use of O-EDF $\overleftrightarrow{\mathscr{V}} \in \mathbb{K}$. In this situation, the membership function of O-EDF $\overleftrightarrow{\mathscr{V}} \in \mathbb{K}$ models the assessment imprecision of a financial instrument.

A rate of return is a function of a future value, which is uncertain. This is due to an investor's lack of certain knowledge about the future. It means that no investor is sure of their future profits or losses. An increase of uncertainty may increase the risk of making an incorrect financial decision. In this paper, we evaluate the uncertainty risk using the oriented expected discount factor. An increase in ambiguity means an increase in the amount of recommended alternative information about the state of affairs. The increase in the ambiguity of O-EDF $\overleftrightarrow{\mathscr{V}} \in \mathbb{K}$ suggests a higher number of alternative recommendations to choose from. This increases the risk of making an incorrect choice from among the recommended alternatives.

This may imply making a decision that will result in less than the maximum profit, which can be understood as a loss of certainty. Ambiguity and uncertainty have the same depiction of the risks they cause. We will assess both risks using different methods because the effects of a decision made both under the risk of ambiguity and under the risk of uncertainty are the same, but their reasons are different. The ambiguity risk loading the oriented fuzzy expected factor $\overleftrightarrow{\mathscr{V}}$ is appraised by the energy measure $d(\overleftrightarrow{\mathscr{V}})$. An increase in the indistinctness of $\overleftrightarrow{\mathscr{V}}$ means that it is more difficult to distinguish between recommended and unrecommended decisions. It implies an increase in the indistinctness risk, that is, the possibility of making an unrecommended decision. The indistinctness risk of O-EDF $\overleftrightarrow{\mathscr{V}}$ is appraised by the entropy measure $e(\overleftrightarrow{\mathscr{V}})$. Imprecision risk includes ambiguity and indistinctness risk. Hence, an increase in uncertainty risk or in imprecision risk worsens the conditions for making a decision. Risk assessment related to uncertainty as to the state of affairs plays a very important role in the decision-making process. Piasecki [67] shown that there exists a possibility of limiting the uncertainty risk by increasing the imprecision risk. Taking indistinctness into account allows us to reject those decision options that have a low cognitive value of the collected information, even if they are attractive from the point of view of the relationship between the expected profits and the assessment of the uncertainty risk.

In our paper, we examined the imprecise security evaluation in the context of uncertainty. The main objective of this article was to analyze the possibility of managing the risk of a portfolio of multiple assets. The portfolio was built using imprecise information describing the present value of the component assets. These imprecise present values were represented by TrOFNs. Relationships between the imprecision risk burdening portfolio components and the same risk burdening multi-assets portfolios have been described. We showed that the proposed portfolio analysis can be fully used for portfolios $\pi^{+}$of rising securities and $\pi^{-}$of falling ones. This allows the investor to manage the portfolio risk since only rising securities can receive a BUY or ACCUMULATE recommendation, while only falling securities can receive a SELL or REDUCE recommendation. We showed that the portfolio diversification can lower uncertainty risk and imprecision risk. All conclusions are compatible with financial practice and theory. Results obtained with the use of the imprecise expected discount factor were applied as input data for the robo-advice systems described in [32]. In further studies, we will compare the methods using trapezoidal fuzzy numbers and trapezoidal oriented fuzzy numbers in portfolio analysis, and we will show that the use of trapezoidal oriented fuzzy numbers is more useful. The aim of further research will also be to investigate the relationship between oriented fuzzy numbers, trapezoidal fuzzy numbers, and intuitionistic fuzzy numbers.

Funding: This research received no external funding.

Institutional Review Board Statement: Not applicable.

Informed Consent Statement: Not applicable. 
Conflicts of Interest: The author declares no conflict of interest.

\section{References}

1. Von Mises, L. The Ultimate Foundation of Economic Science an Essay on Method; D. Van Nostrand Company, Inc.: Princeton, NJ, USA, 1962.

2. Kaplan, S.; Barish, N.N. Decision-Making Allowing Uncertainty of Future Investment Opportunities. Manag. Sci. 1967, 13, B569-B577. [CrossRef]

3. Caplan, B. Probability, common sense, and realism: A reply to Hulsmann and Block. Q. J. Austrian Econ. 2001, 4, 69-86.

4. Czerwiński, Z. Enumerative induction and the theory of games. Studia Log. 1960, 10, 24-36. [CrossRef]

5. Kolmogorov, A.N. Grundbegriffe der Wahrscheinlichkeitsrechnung; Julius Springer: Berlin/Heidelberg, Germany, 1933. [CrossRef]

6. Kolmogorov, A.N. Foundations of the Theory of Probability; Chelsea Publishing Company: New York, NY, USA, 1956.

7. Von Lambalgen, M. Randomness and foundations of probability: Von Mises' axiomatization of random sequences. Inst. Math. Stat. Lect. Notes-Monogr. Ser. 1996, 30, 347-367.

8. Von Mises, R. Probability, Statistics and Truth; The Macmillan Company: New York, NY, USA, 1957.

9. Sadowski, W. Forecasting and decision making. In Quantitative Wirtschafts-und Unternehmensforschung; Henn, R., Schips, B., Stähly, P., Eds.; Springer: Berlin/Heidelberg, Germany, 1980. [CrossRef]

10. Klir, G.J. Developments in uncertainty-based information. Adv. Comput. 1993, 36, 255-332. [CrossRef]

11. Zadeh, L. Fuzzy sets. Inf. Control. 1965, 8, 338-353. [CrossRef]

12. Piasecki, K. Basis of financial arithmetic from the viewpoint of the utility theory. Oper. Res. Decis. 2012, 22, 37-53. [CrossRef]

13. Piasecki, K. Revision of the Kosiński's Theory of Ordered Fuzzy Numbers. Axioms 2018, 7, 16. [CrossRef]

14. Kacprzak, D.; Kosiński, W. Optimizing Firm Inventory Costs as a Fuzzy Problem. Stud. Logic Gramm. Rhetor 2014, 37, 89-105. [CrossRef]

15. Prokopowicz, P.; Czerniak, J.; Mikołajewski, D.; Apiecionek, Ł.; Slezak, D. Theory and Applications of Ordered Fuzzy Number. Tribute to Professor Witold Kosiński; Studies in Fuzziness and Soft Computing, 356; Springer: Berlin/Heidelberg, Germany, 2017. [CrossRef]

16. Kosiński, W.K.; Kosiński, W.; Kościeński, K. Ordered fuzzy numbers approach to an investment project evaluation. Manag. Prod. Eng. Rev. 2013, 4, 50-62. [CrossRef]

17. Piasecki, K.; Łyczkowska-Hanćkowiak, A. On Present Value Evaluation under the Impact of Behavioural Factors Using Oriented Fuzzy Numbers. Symmetry 2021, 13, 468. [CrossRef]

18. Piasecki, K. Expected return rate determined as oriented fuzzy number. In Proceedings of the 35th International Conference Mathematical Methods in Economics Conference, Hradec Králové, Czech Republic, 13-15 September 2017; pp. 561-565.

19. Łyczkowska-Hanćkowiak, A.; Piasecki, K. Two-assets portfolio with trapezoidal oriented fuzzy present values. In Proceedings of the 36th International Conference Mathematical Methods in Economics Conference, Jindřichův Hradec, Czech Republic, 12-14 September 2018; pp. 306-311.

20. Łyczkowska-Hanćkowiak, A. Sharpe's Ratio for Oriented Fuzzy Discount Factor. Mathematics 2019, 7, 272. [CrossRef]

21. Piasecki, K.; Łyczkowska-Hanćkowiak, A. Representation of Japanese Candlesticks by Oriented Fuzzy Numbers. Econometrics 2020, 8, 1. [CrossRef]

22. Łyczkowska-Hanćkowiak, A.; Piasecki, K. The Present Value of a Portfolio of Assets with Present Values Determined by Trapezoidal Ordered Fuzzy Number. Oper. Res. Decis. 2018, 28, 41-56. [CrossRef]

23. Piasecki, K.; Łyczkowska-Hanćkowiak, A. Imprecision Indexes of Oriented Fuzzy Numbers. In Proceedings of the International Workshop on Intuitionistic Fuzzy Sets and General Nets-IWIFSGN 2019, Warsaw, Poland, 24-25 October 2019; Atanassov, K.T., Atanassova, V., Kacprzyk, J., Kałuszko, A., Krawczak, M., Owsiński, J.W., Sotirov, S.S., Sotirova, E., Szmidt, E., Zadrożny, S., Eds.; Artificial Intelligence and Soft Computing 1308; Springer: Cham, Switzerland, 2021; pp. 112-124. [CrossRef]

24. Piasecki, K.; Łyczkowska-Hanćkowiak, A. Heikin-Ashi technique with use of oriented fuzzy numbers. In Proceedings of the International Workshop on Intuitionistic Fuzzy Sets and General Nets IWIFSGN-IWIFSGN 2020, Warsaw, Poland, 10-11 December 2020; Atanassov, K.T., Atanassova, V., Kacprzyk, J., Kałuszko, A., Krawczak, M., Owsiński, J.W., Sotirov, S.S., Sotirova, E., Szmidt, E., Zadrożny, S., Eds.; Artificial Intelligence and Soft Computing. (accepted for publication).

25. Łyczkowska-Hanćkowiak, A.; Piasecki, K. Portfolio discount factor evaluated by oriented fuzzy numbers. In Proceedings of the 39th International Conference Mathematical Methods in Economics MME 2021, Prague, Czech Republic, 8-10 September 2021; Fejfar, J., Hruška, M., Eds.; Czech University of Life Sciences Prague: Praha-Suchdol, Czech Republic, 2021; pp. 299-304.

26. Roszkowska, E.; Kacprzak, D. The fuzzy SAW and fuzzy TOPSIS procedures based on ordered fuzzy numbers. Inf. Sci. 2016, 369, 564-584. [CrossRef]

27. Rudnik, K.; Kacprzak, D. Fuzzy TOPSIS method with ordered fuzzy numbers for flow control in a manufacturing system. Appl. Soft Comput. 2017, 52, 1020-1041. [CrossRef]

28. Piasecki, K.; Roszkowska, E. On application of ordered fuzzy numbers in ranking linguistically evaluated negotiation offers. Adv. Fuzzy Syst. 2018, 2018. [CrossRef]

29. Kacprzak, D. A doubly extended TOPSIS method for group decision making based on ordered fuzzy numbers. Expert Syst. Appl. 2018, 116, 243-254. [CrossRef] 
30. Piasecki, K.; Roszkowska, E.; Łyczkowska-Hanćkowiak, A. Simple Additive Weighting Method Equipped with Fuzzy Ranking of Evaluated Alternatives. Symmetry 2019, 11, 482. [CrossRef]

31. Piasecki, K.; Roszkowska, E.; Łyczkowska-Hanćkowiak, A. Impact of the Orientation of the Ordered Fuzzy Assessment on the Simple Additive Weighted Method. Symmetry 2019, 11, 1104. [CrossRef]

32. Łyczkowska-Hanćkowiak, A. On Application Oriented Fuzzy Numbers for Imprecise Investment Recommendations. Symmetry 2020, 12, 1672. [CrossRef]

33. Piasecki, K.; Roszkowska, E.; Wachowicz, T.; Filipowicz-Chomko, M.; Łyczkowska-Hanćkowiak, A. Fuzzy Representation of Principal's Preferences in Inspire Negotiation Support System. Entropy 2021, 23, 981. [CrossRef]

34. Piasecki, K.; Łyczkowska-Hanćkowiak, A. Oriented Fuzzy Numbers vs. Fuzzy Numbers. Mathematics 2021, 9, 523. [CrossRef]

35. Markowitz, H.S.M. Portfolio Selection. J. Financ. 1952, 7, 77-91. [CrossRef]

36. Li, X.; Quin, Z.; Kar, S. Mean-Variance-Skewness model for portfolio selections with fuzzy returns. Eur. J. Oper. Res. 2010, 202, 239-247. [CrossRef]

37. Quin, Z.; Wen, M.; Gu, C. Mean-absolute deviation portfolio selection model with fuzzy returns. Iran. J. Fuzzy Syst. 2011, 8, 61-75. [CrossRef]

38. Tsaur, R. Fuzzy portfolio model with different investor risk attitudes. Eur. J. Oper. Res. 2013, 227, 385-390. [CrossRef]

39. Tanaka, H.; Guo, P.; Turksen, B. Portfolio selection based on fuzzy probabilities and possibility distributions. Fuzzy Sets Syst. 2000, 111, 387-397. [CrossRef]

40. Duan, L.; Stahlecker, P. A portfolio selection model using fuzzy returns. Fuzzy Optim. Decis. Mak. 2011, 10, 167-191. [CrossRef]

41. Guo, H.; Sun, B.; Karimi, H.R.; Ge, Y.; Jin, W. Fuzzy investment portfolio selection models based on interval analysis approach. Math. Probl. Eng. 2012, 2012. [CrossRef]

42. Gupta, P.; Mittal, G.; Saxena, A. Asset portfolio optimization using fuzzy mathematical programming. Inf. Sci. 2008, 178, 1734-1755. [CrossRef]

43. Gupta, P.; Mittal, G.; Mehlawat, M.K. Multiobjective expected value model for portfolio selection in fuzzy environment. Optim. Lett. 2013, 7, 1765-1791. [CrossRef]

44. Piasecki, K.; Siwek, J. Two-Asset Portfolio with Triangular Fuzzy Present Values-An Alternative Approach. In Contemporary Trends in Accounting, Finance and Financial Institutions. Springer Proceedings in Business and Economics; Choudhry, T., Mizerka, J., Eds.; Springer: Cham, Switzerland, 2018. [CrossRef]

45. Piasecki, K.; Siwek, J. Multi-asset portfolio with trapezoidal fuzzy present values. Stat. Rev. 2018, LXV, 183-199. [CrossRef]

46. Buckley, I.J. The fuzzy mathematics of finance. Fuzzy Sets Syst. 1987, 21, 257-273. [CrossRef]

47. Ward, T.L. Discounted fuzzy cash flow analysis. In Proceedings of the 1985 Fall Industrial Engineering Conference Proceedings; 1985; pp. 476-481.

48. Greenhut, J.G.; Norman, G.; Temponi, C.T. Towards a fuzzy theory of oligopolistic competition. In Proceedings of the IEEE Proceedings of ISUMA-NAFIPS, College Park, MD, USA, 17-20 September 1995; pp. 286-291. [CrossRef]

49. Sheen, J.N. Fuzzy economical analysis of cogeneration project. In Proceedings of the 2004 IEEE Region 10 Conference TENCON 2004, Chiang Mai, Thailand, 21-24 November 2004; Volume 3, pp. 311-314. [CrossRef]

50. Huang, X. Risk curve and fuzzy portfolio selection. Int. J. Prod. Econ. 2007, 106, 1102-1112. [CrossRef]

51. Tsao, C.T. Assessing the probabilistic fuzzy Net Present Value for a capital, Investment choice using fuzzy arithmetic. J. Chin. Inst. Ind. Eng. 2005, 22, 106-118. [CrossRef]

52. Calzi, M.L. Towards a general setting for the fuzzy mathematics of finance. Fuzzy Sets Syst. 1990, 35, 265-280. [CrossRef]

53. Piasecki, K. Behavioural present value. SSRN Electron. J. 2011, 1. [CrossRef]

54. Piasecki, K.; Łyczkowska-Hanćkowiak, A. On Approximation of Any Ordered Fuzzy Number by A Trapezoidal Ordered Fuzzy Number. Symmetry 2018, 10, 526. [CrossRef]

55. Dubois, D.; Prade, H. Operations on fuzzy numbers. Int. J. Syst. Sci. 1978, 9, 613-629. [CrossRef]

56. Delgado, M.; Vila, M.A.; Voxman, W. On a canonical representation of fuzzy numbers. Fuzzy Sets Syst. 1998, 93, 125-135. [CrossRef]

57. Goetschel, R.; Voxman, W. Elementary fuzzy calculus. Fuzzy Sets Syst. 1986, 18, 31-43. [CrossRef]

58. Kosiński, W.; Prokopowicz, P.; Ślęzak, D. Fuzzy numbers with algebraic operations: Algorithmic approach. In Proc. IIS'2002; Klopotek, M., Wierzchoń, S.T., Michalewicz, M., Eds.; Physica: Heidelberg, Germany; Sopot, Poland, 2002; pp. 311-320.

59. De Luca, A.; Termini, S. Entropy and energy measures of fuzzy sets. In Advances in Fuzzy Set Theory and Applications; Gupta, M.M., Ragade, R.K., Yager, R.R., Eds.; North-Holland: Amsterdam, The Netherlands, 1979; pp. 321-338.

60. De Luca, A.; Termini, S. A definition of a nonprobabilistic entropy in the settings of fuzzy set theory. Inform. Control 1972, 20, 301-312. [CrossRef]

61. Piasecki, K. Some remarks on axiomatic definition of entropy measure. J. Intell. Fuzzy Syst. 2017, 33, 1945-1952. [CrossRef]

62. Kosko, B. Fuzzy entropy and conditioning. Inf. Sci. 1986, 40, 165-174. [CrossRef]

63. Pedrycz, W.; Gottwald, S.; Czogała, E. Measures of fuzziness and operations with fuzzy sets. Stochastica 1982, 6, 187-205.

64. Knight, F.H. Risk, Uncertainty, and Profit, Hart, Schaffner \& Marx; Houghton Mifflin Company: Boston, MA, USA, 1921. 
65. Piasecki, K.; Stasiak, M.D. The Forex Trading System for Speculation with Constant Magnitude of Unit Return. Mathematics 2019, 7, 623. [CrossRef]

66. Stirling, W.C. Satisficing Games and Decision Making; Cambridge University Press: Cambridge, UK, 2003. [CrossRef]

67. Piasecki, K. Effectiveness of securities with fuzzy probabilistic return. Oper. Res. Decis. 2011, 21, 65-78. 